\title{
Misannotation Awareness: A Tale of Two Gene-Groups
}

\author{
Tania Nobre $^{1 *}$, M. Doroteia Campos ${ }^{1}$, Eva Lucic-Mercy ${ }^{2}$ and Birgit Arnholdt-Schmitt ${ }^{1}$ \\ ${ }^{1}$ EU Marie Curie Chair, Instituto de Ciências Agrárias e Ambientais Mediterrânicas, Universidade de Évora, Évora, Portugal, \\ ${ }^{2}$ INOQ GmbH, Schnega, Germany
}

Incorrectly or simply not annotated data is largely increasing in most public databases, undoubtedly caused by the rise in sequence data and the more recent boom of genomic projects. Molecular biologists and bioinformaticists should join efforts to tackle this issue. Practical challenges have been experienced when studying the alternative oxidase (AOX) gene family, and hence the motivation for the present work. Commonly used databases were screened for their capacity to distinguish AOX from the plastid terminal oxidase (also called plastoquinol terminal oxidase; PTOX) and we put forward a simple approach, based on amino acids signatures, that unequivocally distinguishes these gene families. Further, available sequence data on the AOX family in plants was carefully revised to: (1) confirm the classification as AOX and (2) identify to which AOX family member they belong

OPEN ACCESS

Edited by:

Richard A. Jorgensen, University of Arizona, USA

Reviewed by: Patrick Finnegan, University of Western Australia, Australia

Nicolas L. Taylor, University of Western Australia, Australia

*Correspondence: Tania Nobre tnobre@uevora.pt

Specialty section:

This article was submitted to Plant Genetics and Genomics, a section of the journal Frontiers in Plant Science

Received: 16 March 2016 Accepted: 02 June 2016

Published: 16 June 2016

Citation:

Nobre T, Campos MD, Lucic-Mercy E and Arnholdt-Schmitt B (2016) Misannotation Awareness: A Tale of Two Gene-Groups.

Front. Plant Sci. 7:868 doi: 10.3389/fp/s.2016.00868 to. We bring forward the urgent need of misannotation awareness and re-annotation of public AOX sequences by highlighting different types of misclassifications and the large under-estimation of data availability.

Keywords: gene family, databases, gene annotation, signature-based classification, phylogeny, alternative oxidase, plastoquinol terminal oxidase

\section{GENERAL BACKGROUND}

It has become clear that the coding portions of the genome are organized hierarchically in gene families and superfamilies. Groups of genes encoding proteins showing similarity with each other, traditionally defined by $>50 \%$ pairwise amino acid similarity (Thornton and Desalle, 2000), are referred to as a gene family, and arise from general duplication or by duplication and shuffling of exons from different genes (partial gene duplication) followed by divergence. In the same way as the nested organization of living organisms, gene families can show great diversity: many families have just a few very similar genes, others encompass a large number of closely related and distant genes and still others have hundreds of almost identical copies. This diversity in family structure reflects their evolutionary history, their function and their regulation. Thorough classification of genes into gene families allows all types of inference about the evolution of genes and their encoded proteins (Thornton and Desalle, 2000; Demuth et al., 2006). The first classification efforts of proteins have been curated experimentally, but the increase in sequence data and the recent boom of genomic data makes it impossible to keep the same level of accuracy in gene family classification (e.g., Frech and Chen, 2010; Riesenfeld and Pollard, 2013). This is a widely recognized challenge, and bioinformaticists have increased research efforts to provide algorithms for accurate automatic classifications (e.g., Moriya et al., 2007; Schnoes et al., 2009; Pedruzzi et al., 2013; Fox et al., 2014; Szklarczyk et al., 2015) and high-throughput functional analysis of large sets of protein sequences (e.g., Roy et al., 2012; Cozzetto et al., 2013; Szklarczyk et al., 2015). However, in parallel with this effort, there is a need for greater awareness of the specialists that produce and use the data 
and of those who can actually change the mis-annotations. Genome annotation is an intrinsically complex process, with many different pipelines that focus mainly on the annotation of protein-coding genes; but all pipelines comprise a homology search step (see Yandell and Ence, 2012). Regardless of how the sequences are obtained (traditional Sanger sequencing or highthroughput) gene identification is mainly inferred by homology, which means that the outcome of the inference is never better than the reference(s) itself. This downstream impact goes beyond the annotation as it affects evolutionary studies and the biological understanding of an organism, as well as analyses of pathways, systems, and metabolic processes (Klimke et al., 2011).

This article intends to bring into light the issue of the incorrectly annotated data through a practical -and by the authors experienced- approach when studying a specific gene family in a taxonomical restrict group, the case study of AOX in plants. It targets primarily molecular plant researchers and other specialists that either work directly with these two gene-groups or are facing similar challenges. They are the ones having the deep knowledge on the gene families that they work with and hence are able to provide the data that bioinformaticists need.

\section{THE AOX FAMILY IN PLANTS-A CASE STUDY}

Alternative oxidase (AOX; EC 1.10.3.11 ubiquinol:O2 oxidoreductase id IPR002680) is a terminal quinol oxidase found in the mitochondria of a wide variety of species from different kingdoms (McDonald, 2008). In plants, it is often present as a small multigene family; e.g., AOX in Arabidopsis is encoded by a multigene family with five members: AOX1a, AOX1b, AOX1c, AOX1d, and AOX2 (Saisho et al., 1997, 2001; Clifton et al., 2006).

Across kingdoms, there is a lack of a general pattern with respect to intron/exon structure in AOX genes (McDonald et al., 2015), and the current view is that AOX arose in prokaryotes and entered the eukaryotic lineage via the primary endosymbiotic event that led to the origin of mitochondria (Finnegan et al., 2003; Atteia et al., 2004; McDonald and Vanlerberghe, 2006). Neimanis et al. (2013) suggest that the evolution to a multigene family might have arisen in plants by a duplication event of a single AOX gene after the separation of the Tracheophyta (vascular plants) from the rest of the Embryophyta. More data is needed to test this hypothesis, which does not exclude the possibility of other gene duplication event $(s)$ or even horizontal gene transfer events, to give rise to the pattern of multigene families as we know it nowadays. Within plants, the most common gene structure for AOX comprises four exons interrupted by three introns, with size conservation for all coding regions except the first exon (Considine et al., 2002; Campos et al., 2009). In higher plants, AOX is encoded by three to five genes distributed in two discrete gene subfamilies termed AOX1 and AOX2 (Whelan et al., 1996; Saisho et al., 1997; Vanlerberghe, 2013). While AOX1 expression is induced by stress stimuli in many tissues and is present in both monocot and eudicot plant species, AOX2 is referred to as being constitutively or developmentally expressed in eudicot species (absent from monocots, probably due to gene loss). Even though this paradigm begins to be challenged (Clifton et al., 2006; Costa et al., 2010; Cavalcanti et al., 2013), the current view is still that the subfamilies have different physiological roles. They are thus expected to have evolved under different selection pressures. Costa et al. (2014) have produced the most recent classification scheme of AOX in Angiosperms, and they show that there is an urgent need to reannotate previously named AOXs. This effort needs to be expanded for plants in general and, ideally, for AOX in all kingdoms. With the present work, we call for awareness on careful annotation of public AOX sequences by highlighting misclassifications at different levels.

\section{AOX vs. PTOX, Both Membrane-Bound di-Iron Carboxylate Proteins}

AOX, a non-proton motive ubiquinol oxidase, belongs to the di-iron carboxylate protein superfamily which includes members that are soluble in the cytosol and members that are membrane-bound. AOX and plastid terminal oxidase (PTOX; EC 1.10.3.11 ubiquinol:O2 oxidoreductase id IPR002680) are the membrane-bound members of this superfamily. Whereas AOX is present in almost all phyla, PTOX appears limited to organisms capable of oxygenic photosynthesis (McDonald and Vanlerberghe, 2006). Both are quinol oxidases, but the first is located in the mitochondrial inner membrane at the mitochondria, is involved in the respiratory electron transport and interacts with ubiquinol; the second locates in the stroma, is active in the photosynthetic electron transport and it catalyzes the oxidation with plastoquinol (on AOX role see Vanlerberghe, 2013; on PTOX role see Nawrocki et al., 2015; KriegerLiszkay and Feilke, 2016). Often, these two family members are mis-annotated in sequence databases.

In a quick survey on sequences deposited at NCBI (using a known AOX sequence and the BLAST tool) at least seven protein sequences, from either gene-discovery sequencing projects or gene re-sequencing, and six sequences originating from whole genome analysis, are annotated as AOX but they are indeed PTOX (Supplementary Data 1). Still other sequences are annotated as immutans, often used as a synonym for PTOX (probably because the immutans variegation mutant of Arabidopsis does not have a fully functional PTOX; Wu et al., 1999). Yet, another nine entries that correspond to NCBI annotations appear as alternative oxidase 4 or ubiquinol oxidase 4. Not all this annotations are actually wrong (for instances, the use of immutans to refer to PTOX), but the non-conformity of the nomenclature only raises confusion and often is the cause of the propagation of error. See for example the accession ABD32645 from 2006, a protein sequence from Medicago truncatula annotated as encoding for the product alternative oxidase (although it belongs to PTOX, see reasons below). This was spread to more recent data originated from Medicago genome sequencing (see accession AES64415) because both traditional sequencing, and certainly genome sequencing projects, use as reference previously annotated sequences.

How to proceed then? There are several compelling reasons to use deduced protein sequences, rather than nucleotide 
sequences, to determine gene identification for annotation purposes (including the availability of good and maintained databases of protein sequences: e.g., Mulder and Apweiler, 2002; Hunter et al., 2009; Sigrist et al., 2013; Finn et al., 2014). Homology searches based on protein sequences lowers the signalto-noise ratio in sequence searches and alignments, and eases the distinction of true homology from random similarity because of the close relation between protein sequence and function. Protein sequences can be analyzed fully, but to rapidly define the family of proteins to which the sequence belongs to it is practical to look for single domains or motifs. These motifs have been shown to be important for protein functionality and can outline a family of proteins (Martinez, 2013), being thus useful for implementation in protein classification systems. The large influx of raw sequence data from genome sequencing projects has led to the emergence of numerous automatic methods for protein sequence analysis and classification, based on comparative analyses. These bioinformatic tools largely rely on the identification of motifs that could be previously encountered in characterized protein families. Two main types of databases are available, (1) the traditional gene family databases trusting on signatures such as Pfam (Finn et al., 2014) or PROSITE (Sigrist et al., 2013) and (2) the integrative databases such as InterPro (Hunter et al., 2009) and CDD (Marchler-Bauer et al., 2011) joining information from several major signature databases (Martinez, 2011, 2013). Signature-based methods are routinely used for gene function annotation but they seem to have a limitation in the case of distinguishing between AOX/PTOX: whereas all databases return sequences with high homologies to the sequence of interest, their classification is not consensual and most fail to clearly distinguish these two sub-families (Supplementary Table 1).

Actually, a multiple sequence alignment of full-length amino acid sequences of the putative gene models, as well as characterized sequences, followed by a neighbor-joining clustering showed that AOX and PTOX form clearly different clades (Figure 1A). We have found two sites that seem to show a specific conservation of amino acids related to the subfamily members-fingerprints-and we propose their use as a quick and efficient way to distinguish plant AOX from PTOX sequences. These signatures may serve as identification motifs specific for the two subfamilies, and when scanned against the GenBank database, retrieved only members of each subfamily. These sequences can be used to identify additional members of the two subfamilies in other plant species as their genomes are being sequenced. The general structure of both AOX and PTOX is different and on itself might represent an extra way to verify gene affiliation of already published sequences to either AOX or PTOX family member (Figure 1B).

\section{Members of the AOX Family}

The naming of AOX genes originally occurred in the order of their discovery in a species but the need of a classification system became obvious as more sequences were made available. Considine et al. (2002) provided an initial classification that divided plant AOX in two subfamilies (AOX1 and AOX2) while in non-plant species the AOX was named as AOX0. Recently, Costa et al. (2014) proposed a classification scheme for AOX in angiosperms based on protein tree topologies, the analysis of specific amino acid sites found to differ between AOX subfamilies and subtypes, and the known evolutionary history of angiosperms.

The protein alignments made available by Costa et al. (2014) as Supplementary Material were used as the base data for our phylogenetic analysis. To the dataset, other sequences retrieved from the different databases were added including the few existing data on Gymnosperms, giving a total of 369 sequences. The sequences retrieved in this work were analyzed for the presence/absence of the specific amino acid motifs and a tentative AOX classification was constructed (Supplementary Table 2). Most of those sequences were obtained from genome sequencing projects and were often not annotated. Adding to the 32 mis-annotated sequences found by Costa et al. (2014), we found 13 more mis-annotated sequences (in 120 sequences, of which 73 were without any annotation and 22 were referred to simply as AOX). Altogether, 369 sequences were used on the phylogenetic analysis (Figure 2; see Supplementary Data 3) and their classification was adjusted. The phylogeny returned is largely unresolved, suggesting an almost simultaneous divergence from a common ancestor, or simply a lack of information to resolve the polytomies. These polytomies can also be the result of convergent evolution and/or of recent gene duplication (both previously suggested for AOX, e.g., Neimanis et al., 2013; Costa et al., 2014). Classification and annotation can be further hindered by the observation that some plants that are more phylogenetically divergent have similar multigene families representatives (e.g., Arabidopsis and poplar, a Brassicales and a Malpighiales, both have AOX1a, AOX1b, AOX1c, and AOX1d), while more closely related species have large differences in AOX family composition (e.g., Arabidopsis and papaya, both Brassicales, the first with all known members of AOX1 and the last with just one AOX1 gene; Costa et al., 2014; Cardoso et al., 2015).

\section{Short Note on Gene Isolation and Annotation}

When starting the isolation process of a specific gene in a species where no further information is available, the most common laboratory strategy has been the use of "universal primers" (when existing). In the case of AOX, the most commonly used primers are the ones described by Saisho et al. (1997). These seem to work well across kingdoms and, given what we know about the evolutionary story of AOXs, also across family members. After database searches for DNA sequences encoding for the different AOX genes and an in silico amplification of the region comprised between the primers P1 and P2 as probes (Saisho et al., 1997), we have got 218 sequences of the expected 444 bp amplicon (with no insertions or deletions; Supplementary Data 4). The resulting NJ tree highlights that, with this conserved fragment, it is possible to discriminate between AOX1 and AOX2 but no other gene member can be clearly identified (Supplementary Figure 1) and should thus not be further annotated only on the basis of fragment homology. 


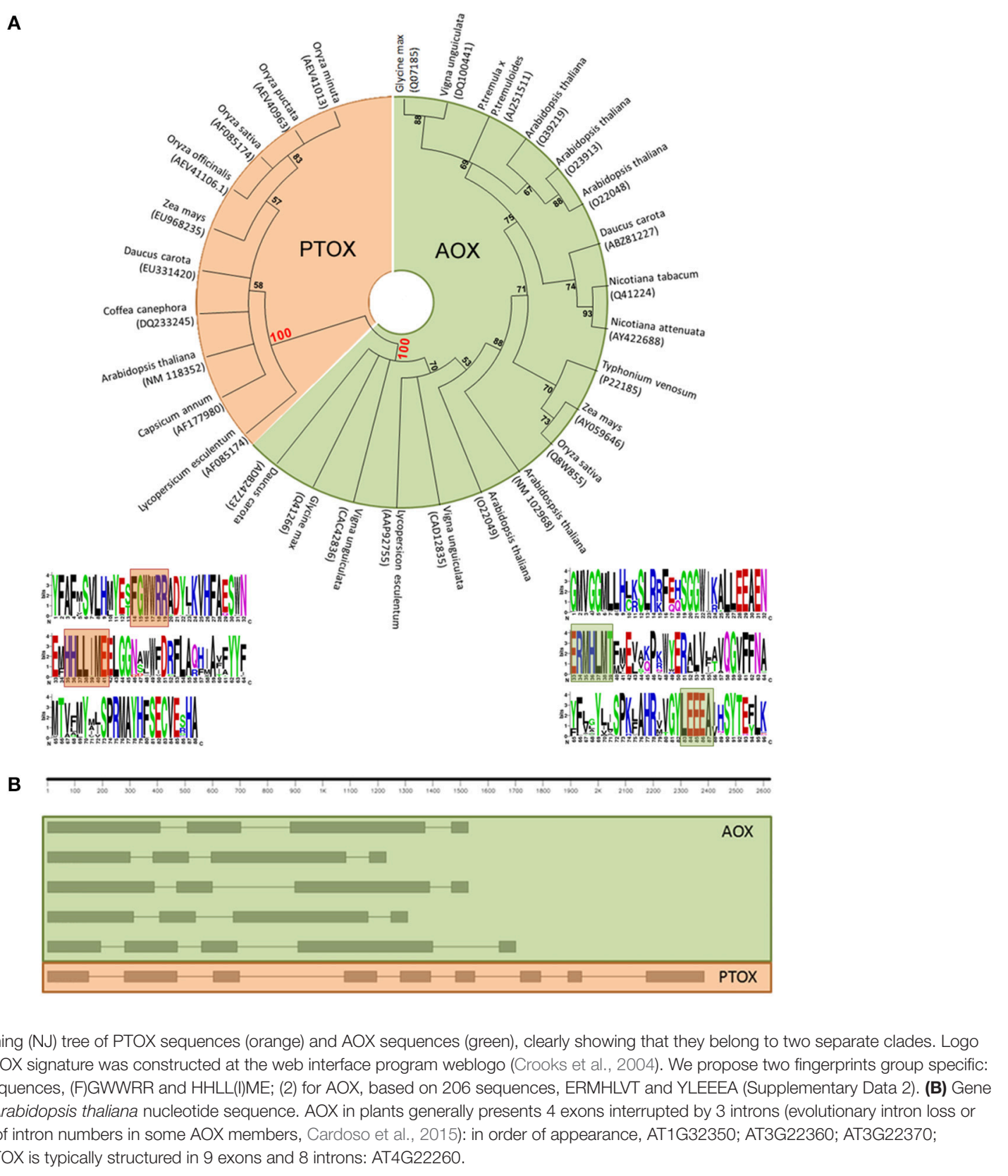

\section{FINAL CONSIDERATIONS}

Focusing on the membrane-bound di-iron carboxylate proteins, we show that a re-annotation is needed. We have reviewed a large quantity of data present in different databases, identified an easy way (based on signatures) of distinguishing between the two mis-classified gene families-AOX vs. PTOX - and presented the largest phylogeny to date that comprises curated and annotated AOX amino acids sequences (based on the system developed by Costa et al., 2014) as well as newly identified sequences from genomic databases. In the particular case of AOX, we realize that the great majority of data available is "hidden" in contigs and scaffoldings as non-identified regions. If at one side this implies that there are fewer non-spotted misannotations than originally thought, it also means that the amount of data available for this gene family is largely under-estimated.

Misannotation in superfamilies containing multiple families that catalyze different reactions is an issue needing to be addressed as it can have serious repercussions on data interpretation and ultimately on our understanding of the systems. It is a shared responsibility, of researchers working with these superfamilies and bioinformaticists to tackle this challenge. The current identification and classification system is prone to error propagation and an increase in annotation errors over 

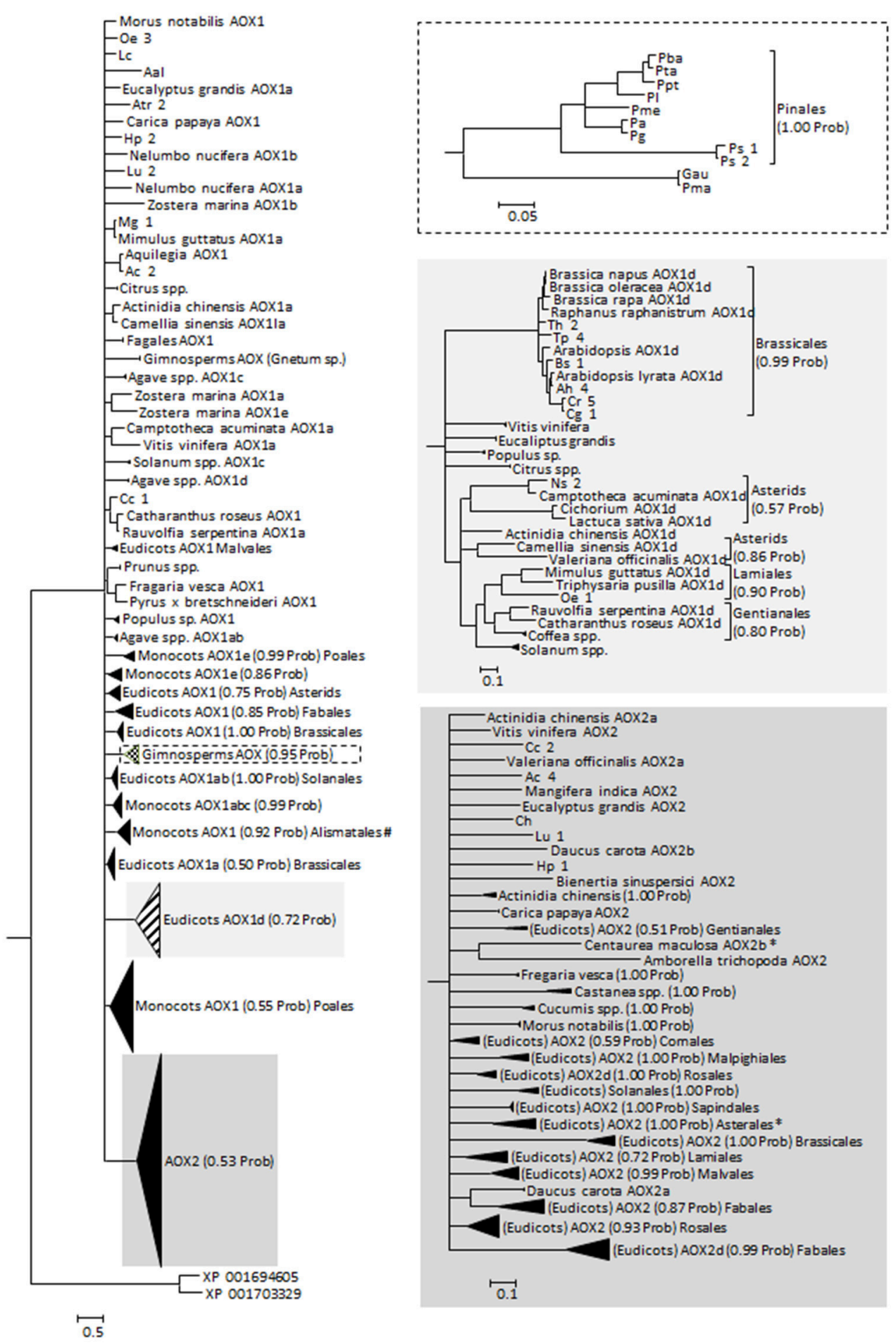

FIGURE 2 | Reconstructed phylogeny of plants at the gene family AOX included in this study (a combination of newly collected sequences and the alignment made available by Costa et al., 2014). The optimal substitution model was selected in MrModeltest 2.2 (Posada and Crandall, 1998) as being the $\mathrm{JTT}+\mathrm{I}+\mathrm{G}$. The phylogeny corresponds to the majority rule consensus tree of trees sampled in a Bayesian analysis (conducted using MrBayes version 3.0 (Huelsenbeck and Ronquist, 2001; Ronquist and Huelsenbeck, 2003); with default settings and with MCMC-considering 100, 000 generations -runs being repeated three times as a safeguard against spurious results; first 1000 trees were discarded as burn-in; stationarity was confirmed by analysis of the log-likelihoods and the consistency between runs). The numbers above the branches refer to the Bayesian posterior probability of the nodes (more than 50\%) derived from 19500 Markov chain Monte Carlo-sampled trees. \#Clade containing a putative Solanum tuberosum AOX1 sequence (sequence id PRF: 1588565); likely a misidentification of the organism. ${ }^{*}$ Centaurea maculosa (Asterales) AOX2b sequence (GenBank EH723572.1) does not cluster with the other Asterales. 
time is to be expected. Researcher's awareness can minimize error propagation and certainly errors in data interpretation. It is also a dynamic process that needs to be revised and updated in frame of the continuous new flow of data. The ones with deeper knowledge on the specific genes families can positively contribute to data revision and collaborate with bioinformaticists to the updating of the classification systems.

\section{AUTHOR CONTRIBUTIONS}

TN and BA-S conceptualized the manuscript. TN, MDC, and EL-M collected and analyzed the data. TN designed and wrote the manuscript and all the authors revised and approved it.

\section{ACKNOWLEDGMENTS}

The authors would like to thank the reviewers, for their constructive critics, Hélio Costa, for the discussions during the preparation of this manuscript and Catarina Campos, for revision of a previous version of this manuscript. TN

\section{REFERENCES}

Atteia, A., van Lis, R., van Hellemond, J. J., Tielens, A. G., Martin, W., and Henze, K. (2004). Identification of prokaryotic homologues indicates an endosymbiotic origin for the alternative oxidases of mitochondria (AOX) and chloroplasts (PTOX). Gene 330, 143-148. doi: 10.1016/j.gene.2004.0 1.015

Campos, M. D., Cardoso, H. G., Linke, B., Costa, J. H., de Melo, D. F., Justo, L., et al. (2009). Differential expression and co-regulation of carrot AOX genes (Daucus carota). Physiol. Plant. 137, 578-591. doi: 10.1111/j.1399-3054.2009.01 282.x

Cardoso, H. G., Nogales, A., Frederico, A. M., Svensson, J. T., Macedo, E. S., Valadas, V., et al. (2015). "Exploring AOX gene diversity," in Alternative Respiratory Pathways in Higher Plants, eds K. J. Gupta, L. A. J. Mur, and B. Neelwarne (Chichester: John Wiley \& Sons, Ltd.), 239-254.

Cavalcanti, J. H. F., Oliveira, G. M., Saraiva, K. D. D. C., Torquato, J. P. P., Maia, I. G., de Melo, D. F., et al. (2013). Identification of duplicated and stress-inducible Aox $2 \mathrm{~b}$ gene co-expressed with Aox 1 in species of the Medicago genus reveals a regulation linked to gene rearrangement in leguminous genomes. J. Plant Physiol. 170, 1609-1619. doi: 10.1016/j.jplph.2013.06.012

Clifton, R., Millar, A. H., and Whelan, J. (2006). Alternative oxidases in Arabidopsis: a comparative analysis of differential expression in the gene family provides new insights into function of non-phosphorylating bypasses. Biochim. Biophys. Acta 1757, 730-741. doi: 10.1016/j.bbabio.2006.03.009

Considine, M. J., Holtzapffel, R. C., Day, D. A., Whelan, J., and Millar, A. H. (2002). Molecular distinction between alternative oxidase from monocots and dicots 1 . Plant Physiol. 129, 949-953. doi: 10.1104/pp.004150.isozymes

Costa, J. H., McDonald, A. E., Arnholdt-Schmitt, B., and Fernandes de Melo, D. (2014). A classification scheme for alternative oxidases reveals the taxonomic distribution and evolutionary history of the enzyme in angiosperms. Mitochondrion 19, 172-183. doi: 10.1016/j.mito.2014.04.007

Costa, J. H., Mota, E. F., Cambursano, M. V., Lauxmann, M. A., de Oliveira, L. M. N., Silva Lima, M. D. G., et al. (2010). Stress-induced co-expression of two alternative oxidase (VuAox1 and 2b) genes in Vigna unguiculata. J. Plant Physiol. 167, 561-570. doi: 10.1016/j.jplph.2009.11.001

Cozzetto, D., Buchan, D. W., Bryson, K., and Jones, D. T. (2013). Protein function prediction by massive integration of evolutionary analyses and multiple data sources. BMC Bioinformatics 14:S1. doi: 10.1186/1471-2105-14-S3-S1

Crooks, G., Hon, G., Chandonia, J., and Brenner, S. (2004). NCBI GenBank FTP Site $\backslash \mathrm{n}$ WebLogo: a sequence logo generator. Genome Res. 14, 1188-1190. doi: $10.1101 /$ gr.849004.1 was supported by a Marie Curie Career Integration grant (FP7-PEOPLE-2012-CIG ref. 321725) and a Portuguese FCTFoundation for Science and Technology (FCT) fellowship (SFRH/BCC/52187/2013). MDC also thanks FCT for the support given through a PhD fellowship (SFRH/BD/65354/2009). EL-M appreciates the support given by AGRO-AMF-AOX project (Industry-Academia Partnerships and Pathways-IAPP-FP7) in form of a Marie Curie Fellowship for a secondment to the Portuguese group. BA-S appreciates funding by the European Commission of both CIG and IAPP projects, FCT grants and support given through the University of Évora. This work is funded by National Funds through FCT-Foundation for Science and Technology under the Project UID/AGR/ $00115 / 2013$.

\section{SUPPLEMENTARY MATERIAL}

The Supplementary Material for this article can be found online at: http://journal.frontiersin.org/article/10.3389/fpls.2016. 00868

Demuth, J. P., De Bie, T., Stajich, J. E., Cristianini, N., and Hahn, M. W. (2006). The evolution of mammalian gene families. PLOS ONE 1:e85. doi: 10.1371/journal.pone.0000085

Finn, R. D., Bateman, A., Clements, J., Coggill, P., Eberhardt, R. Y., Eddy, S. R., et al. (2014). Pfam: the protein families database. Nucleic Acids Res. 42, D222-D230. doi: 10.1093/nar/gkt1223

Finnegan, P. M., Umbach, A. L., and Wilce, J. A. (2003). Prokaryotic origins for the mitochondrial alternative oxidase and plastid terminal oxidase nuclear genes. FEBS Lett. 555, 425-430. doi: 10.1016/S0014-5793(03)01309-7

Fox, N. K., Brenner, S. E., and Chandonia, J. M. (2014). SCOPe: structural classification of proteins - extended, integrating SCOP and ASTRAL data and classification of new structures. Nucleic Acids Res. 42, 304-309. doi: 10.1093/nar/gkt1240

Frech, C., and Chen, N. (2010). Genome-wide comparative gene family classification. PLoS ONE 5:e13409. doi: 10.1371/journal.pone.0013409

Huelsenbeck, J. P., and Ronquist, F. (2001). MRBAYES: Bayesian inference of phylogenetic trees. Bioinformatics 17, 754-755. doi: 10.1093/bioinformatics/17. 8.754

Hunter, S., Apweiler, R., Attwood, T. K., Bairoch, A., Bateman, A., Binns, D., et al. (2009). InterPro: the integrative protein signature database. Nucleic Acids Res. 37, D211-D215. doi: 10.1093/nar/gkn785

Klimke, W., O’Donovan, C., White, O., Brister, J. R., Clark, K., Fedorov, B., et al. (2011). Solving the problem: genome annotation standards before the data deluge. Stand. Genomic Sci. 5, 168-193. doi: 10.4056/sigs.2084864

Krieger-Liszkay, A., and Feilke, K. (2016). The dual role of the plastid terminal oxidase PTOX: between a protective and a pro-oxidant function. Front. Plant Sci. 6:1147. doi: 10.3389/fpls.2015.01147

Marchler-Bauer, A., Lu, S., Anderson, J. B., Chitsaz, F., Derbyshire, M. K., DeWeese-Scott, C., et al. (2011). CDD: a Conserved Domain Database for the functional annotation of proteins. Nucleic Acids Res. 39, D225-D229. doi: 10.1093/nar/gkq1189

Martinez, M. (2011). Plant protein-coding gene families: emerging bioinformatics approaches. Trends Plant Sci. 16, 558-567. doi: 10.1016/j.tplants.2011.06.003

Martinez, M. (2013). From plant genomes to protein families: computational tools. Comput. Struct. Biotechnol. J. 8, 1-5. doi: 10.5936/csbj.201307001

McDonald, A. E. (2008). Alternative oxidase: an inter-kingdom perspective on the function and regulation of this broadly distributed "cyanide-resistant" terminal oxidase. Funct. Plant Biol. 35, 535-552. doi: 10.1071/FP08025

McDonald, A. E., Costa, J. H., Nobre, T., de Melo, D. F., and Arnholdt-Schmitt, B. (2015). "Evolution of AOX genes across kingdoms and the challenge of classification," in Alternative Respiratory Pathways in Higher Plants, eds K. J. 
Gupta, L. A. J. Mur, and B. Neelwarne (Chichester: John Wiley \& Sons, Ltd.), 267-272.

McDonald, A. E., and Vanlerberghe, G. C. (2006). Origins, evolutionary history, and taxonomic distribution of alternative oxidase and plastoquinol terminal oxidase. Comp. Biochem. Physiol. Part D Genomics Proteomics 1, 357-364. doi: 10.1016/j.cbd.2006.08.001

Moriya, Y., Itoh, M., Okuda, S., Yoshizawa, A. C., and Kanehisa, M. (2007). KAAS: an automatic genome annotation and pathway reconstruction server. Nucleic Acids Res. 35, 182-185. doi: 10.1093/nar/gkm321

Mulder, N. J., and Apweiler, R. (2002). Tools and resources for identifying protein families, domains and motifs. Genome Biol. 3, reviews2001.1-reviews2001.8. doi: 10.1186/gb-2001-3-1-reviews2001

Nawrocki, W. J., Tourasse, N. J., Taly, A., Rappaport, F., and Wollman, F.-A. (2015). The Plastid terminal oxidase: its elusive function points to multiple contributions to plastid physiology. Annu. Rev. Plant Biol. 66, 49-74. doi: 10.1146/annurev-arplant-043014-114744

Neimanis, K., Staples, J. F., Hüner, N. P. A., and Mcdonald, A. E. (2013). Identification, expression, and taxonomic distribution of alternative oxidases in non-angiosperm plants. Gene 526, 275-286. doi: 10.1016/j.gene.2013.0 4.072

Pedruzzi, I., Rivoire, C., Auchincloss, A. H., Coudert, E., Keller, G., De Castro, E., et al. (2013). HAMAP in 2013, new developments in the protein family classification and annotation system. Nucleic Acids Res. 41, 584-589. doi: 10.1093/nar/gks1157

Posada, D., and Crandall, K. A. (1998). Bioinformatics application note MODELTEST: testing the model of DNA substitution. Bioinformatics 14, 817-818.

Riesenfeld, S. J., and Pollard, K. S. (2013). Beyond classification: gene-family phylogenies from shotgun metagenomic reads enable accurate community analysis. BMC Genomics 14:419. doi: 10.1186/1471-2164-14-419

Ronquist, F., and Huelsenbeck, J. P. (2003). MrBayes 3: Bayesian phylogenetic inference under mixed models. Bioinformatics 19, 1572-1574. doi: 10.1093/bioinformatics/btg180

Roy, A., Yang, J., and Zhang, Y. (2012). COFACTOR: an accurate comparative algorithm for structure-based protein function annotation. Nucleic Acids Res. 40, 1-7. doi: 10.1093/nar/gks372

Saisho, D., Nakazono, M., Lee, K.-H., Tsutsumi, N., Akita, S., and Hirai, A. (2001). The gene for alternative oxidase-2 (AOX2) from Arabidopsis thaliana consists of five exons unlike other AOX genes and is transcribed at an early stage during germination. Genes Genet. Syst. 76, 89-97. doi: 10.1266/ggs.76.89
Saisho, D., Nambara, E., Naito, S., Tsutsumi, N., Hirai, A., and Nakazono, M. (1997). Characterization of the gene family for alternative oxidase from Arabidopsis thaliana. Plant Mol. Biol. 35, 585-596.

Schnoes, A. M., Brown, S. D., Dodevski, I., and Babbitt, P. C. (2009). Annotation error in public databases: misannotation of molecular function in enzyme superfamilies. PLoS Comput. Biol. 5:e1000605. doi: 10.1371/journal.pcbi.1000605

Sigrist, C. J., de Castro, E., Cerutti, L., Cuche, B. A., Hulo, N., Bridge, A., et al. (2013). New and continuing developments at PROSITE. Nucleic Acids Res. 41, D344-D347. doi: 10.1093/nar/gks1067

Szklarczyk, D., Franceschini, A., Wyder, S., Forslund, K., Heller, D., HuertaCepas, J., et al. (2015). STRING v10: protein-protein interaction networks, integrated over the tree of life. Nucleic Acids Res. 43, D447-D452. doi: 10.1093/nar/gku1003

Thornton, J. W., and Desalle, R. (2000). Gene family evolution and homology. Annu. Rev. Genomics Hum. Genet. 1, 41-73. doi: 10.1146/annurev.genom. 1.1 .41

Vanlerberghe, G. C. (2013). Alternative Oxidase: a mitochondrial respiratory pathway to maintain metabolic and signaling homeostasis during abiotic and biotic stress in plants. Int. J. Mol. Sci. 14, 6805-6847. doi: 10.3390/ijms14046805

Whelan, J., Millar, A. H., and Day, D. A. (1996). The alternative oxidase is encoded in a multigene family in soybean. Planta 61, 197-201.

Wu, D., Wright, D. A., Wetzel, C., Voytas, D. F., and Rodermel, S. (1999) The IMMUTANS variegation locus of Arabidopsis defines a mitochondrial alternative oxidase homolog that functions during early chloroplast biogenesis. Plant Cell 11, 43-55. doi: 10.1105/tpc.11.1.43

Yandell, M., and Ence, D. (2012). A beginner's guide to eukaryotic genome annotation. Nat. Rev. Genet. 13, 329-342. doi: 10.1038/nrg3174

Conflict of Interest Statement: The authors declare that the research was conducted in the absence of any commercial or financial relationships that could be construed as a potential conflict of interest.

Copyright (C) 2016 Nobre, Campos, Lucic-Mercy and Arnholdt-Schmitt. This is an open-access article distributed under the terms of the Creative Commons Attribution License (CC BY). The use, distribution or reproduction in other forums is permitted, provided the original author(s) or licensor are credited and that the original publication in this journal is cited, in accordance with accepted academic practice. No use, distribution or reproduction is permitted which does not comply with these terms. 